\title{
Erratum: Revised constraints and Belle II sensitivity for visible and invisible axion-like particles
}

\author{
Matthew J. Dolan, ${ }^{a}$ Torben Ferber, ${ }^{b}$ Christopher Hearty, ${ }^{b, c}$ Felix Kahlhoefer ${ }^{d, e}$ \\ and Kai Schmidt-Hoberg ${ }^{d}$ \\ ${ }^{a}$ ARC Centre of Excellence for Particle Physics at the Terascale, \\ School of Physics, University of Melbourne, \\ 3010, Australia \\ ${ }^{b}$ Department of Physics and Astronomy, University of British Columbia, \\ Vancouver, British Columbia, V6T 1 Z1 Canada \\ ${ }^{c}$ Institute of Particle Physics, \\ Vancouver, British Columbia, V6T $1 Z 1$ Canada \\ ${ }^{d} D E S Y$, \\ Notkestrasse 85, D-22607 Hamburg, Germany \\ ${ }^{e}$ Institute for Theoretical Particle Physics and Cosmology (TTK), RWTH Aachen University, \\ D-52056 Aachen, Germany \\ E-mail: dolan@unimelb.edu.au, ferber@physics.ubc.ca, \\ hearty@physics.ubc.ca, felix.kahlhoefer@desy.de, \\ kai.schmidt.hoberg@desy.de
}

ERRATUM TO: JHEP12(2017)094

ArXiv ePrint: 1709.00009

A mistake has been found in the numerical code used to reproduce the bounds from proton beam dump experiments from ref. [1] in figures 2 and 7 of ref. [2]. Correcting this mistake leads to slightly stronger bounds as shown below. We note that this correction does not include recent improvements in the analysis of proton beam dump experiments [3]. Additional recent bounds on $\mathrm{GeV}$-scale ALPs can be found in refs. [4-8].

\section{Acknowledgments}

We thank Alon Hershenhorn for bringing the mistake to our attention. 

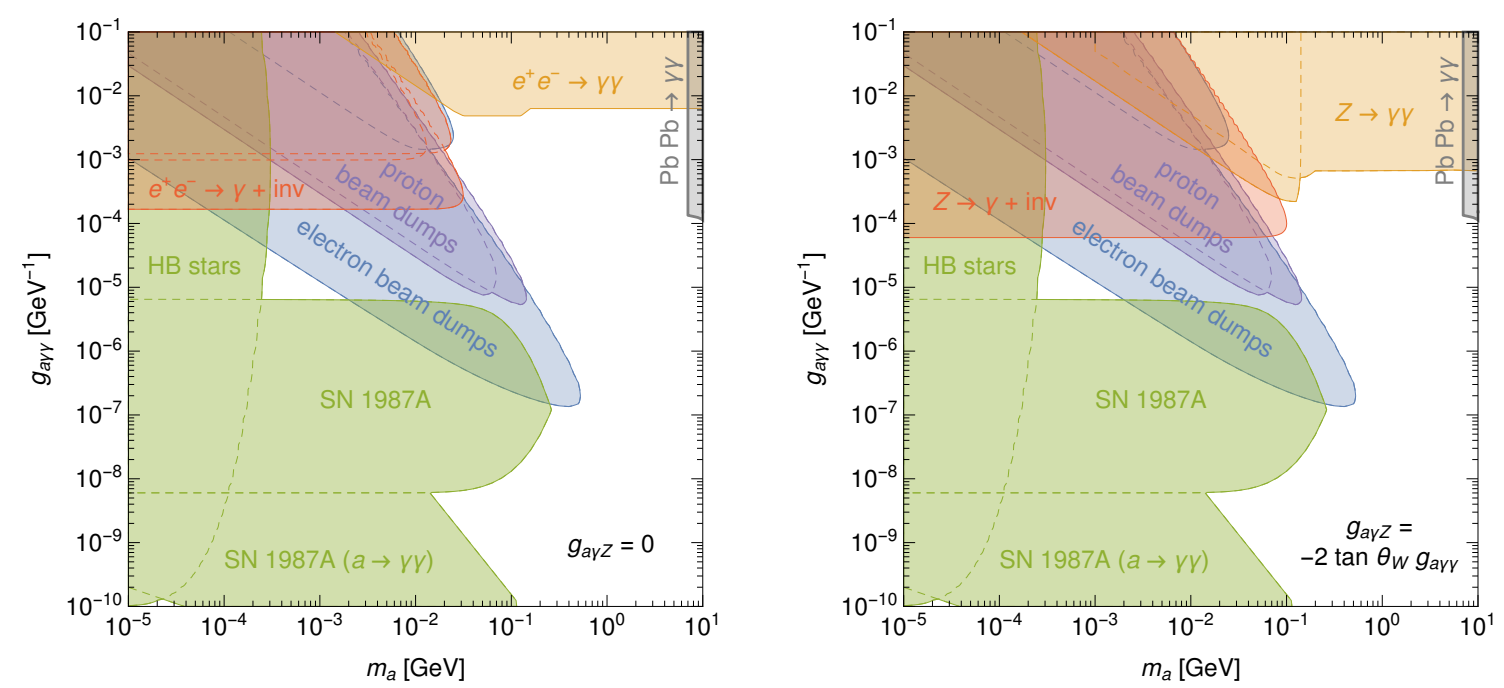

Figure 1. Corrected constraints on ALPs with photon coupling (left) and hypercharge coupling (right). This figure supersedes figure 2 of ref. [2].
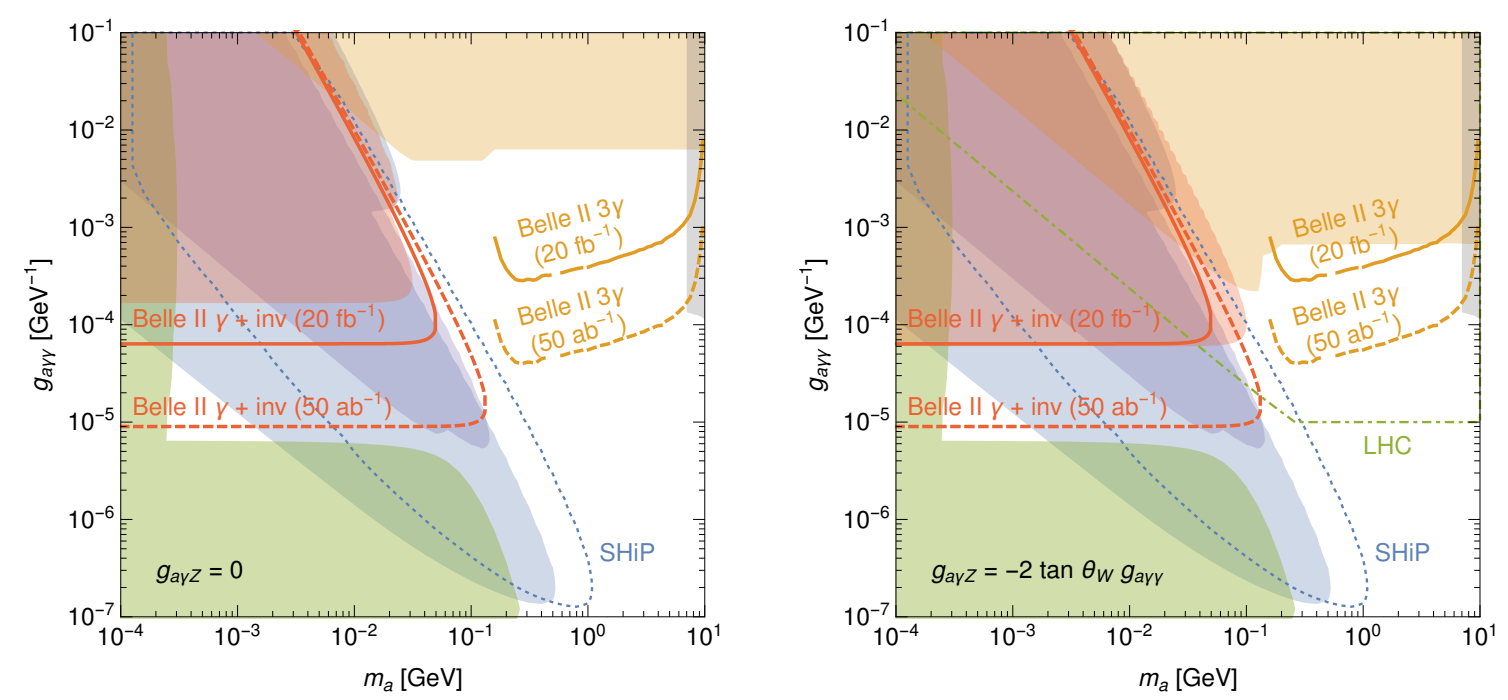

Figure 2. Projected Belle II sensitivity (90\% CL) compared to existing constraints on ALPs with photon coupling (left) and hypercharge coupling (right). This figure supersedes figure 7 of ref. [2].

Open Access. This article is distributed under the terms of the Creative Commons Attribution License (CC-BY 4.0), which permits any use, distribution and reproduction in any medium, provided the original author(s) and source are credited.

\section{References}

[1] B. Döbrich, J. Jaeckel, F. Kahlhoefer, A. Ringwald and K. Schmidt-Hoberg, ALPtraum: ALP production in proton beam dump experiments, JHEP 02 (2016) 018 [arXiv:1512.03069] [INSPIRE]. 
[2] M.J. Dolan, T. Ferber, C. Hearty, F. Kahlhoefer and K. Schmidt-Hoberg, Revised constraints and Belle II sensitivity for visible and invisible axion-like particles, JHEP 12 (2017) 094 [arXiv: 1709.00009] [INSPIRE].

[3] B. Döbrich, J. Jaeckel and T. Spadaro, Light in the beam dump - ALP production from decay photons in proton beam-dumps, JHEP 05 (2019) 213 [Erratum ibid. 10 (2020) 046] [arXiv:1904.02091] [INSPIRE].

[4] S. Knapen, T. Lin, H.K. Lou and T. Melia, Searching for Axionlike Particles with Ultraperipheral Heavy-Ion Collisions, Phys. Rev. Lett. 118 (2017) 171801 [arXiv:1607.06083] [INSPIRE].

[5] CMS collaboration, Evidence for light-by-light scattering and searches for axion-like particles in ultraperipheral $\mathrm{PbPb}$ collisions at $\sqrt{s_{\mathrm{NN}}}=5.02 \mathrm{TeV}$, Phys. Lett. B 797 (2019) 134826 [arXiv: 1810.0460$]$.

[6] D. Aloni, C. Fanelli, Y. Soreq and M. Williams, Photoproduction of Axionlike Particles, Phys. Rev. Lett. 123 (2019) 071801 [arXiv: 1903.03586] [InSPIRE].

[7] NA64 collaboration, Search for Axionlike and Scalar Particles with the NA64 Experiment, Phys. Rev. Lett. 125 (2020) 081801 [arXiv: 2005. 02710] [INSPIRE].

[8] Belle-II collaboration, Search for Axion-Like Particles produced in $e^{+} e^{-}$collisions at Belle II, Phys. Rev. Lett. 125 (2020) 161806 [arXiv: 2007.13071] [InSPIRE]. 